\title{
Studia
}

\section{La réserve héréditaire dans le règlement 650/2012 sur les successions}

\begin{abstract}
The article addresses the issues relating to the protection of forced heirs in international context with a particular focus on the provisions of the EU Succession Regulation pertaining thereto. It contrasts common law tradition with the solutions adopted in French law, whereby certain relatives are entitled to the hereditary reserve ( $l a$ réserve héréditaire). The author discusses selected examples taken from a body of French case-law dealing with the issue in question. Amongst the cases touched upon by the author are those concerning the successions of Johnny Hallyday and Maurice Jarre, which were two cases widely discussed in the recent French jurisprudence.
\end{abstract}

Keywords: forced heirship rights — forced heirs — EU Succession Regulation — hereditary reserve - public policy exception — private international law

La réserve héréditaire est la portion de succession réservée par la loi à certains héritiers (les réservataires). Cette portion ne peut, à peine de réduction, être entamée par des libéralités que le défunt aurait consenties au détriment des réservataires. Le défunt ne peut disposer à titre gratuit que de la quotité disponible.

Cette institution reposait à l'origine sur l'idée de copropriété familiale et elle a évolué vers l'idée de protection des proches du défunt, descendants, ascendants et conjoint. Très étendue dans certains droits, comme en droit français (réserve des 3/4 de la succession quand le défunt laisse

\footnotetext{
a) Prof., Université Paris I Panthéon-Sorbonne.
} 
trois enfants ou davantage), elle est plus limitée dans d'autres, comme en droit allemand et, apparemment, aussi en droit polonais, où la réserve est généralement égale à la moitié de la part légale du réservataire. En revanche, dans les pays de common law, comme l'Angleterre et de nombreux États des États-Unis comme New York et la Californie, la réserve est inconnue, mais les membres de la famille et les personnes à charge peuvent demander au tribunal un soutien financier qui sera prélevé sur les biens de la succession.

Cette diversité législative pose évidemment dans les situations à caractère international un certain nombre de questions, dont celle de la loi applicable. Le règlement européen $n^{\circ} 650 / 2012$ du 4 juillet 2012 a essayé de les résoudre. Rompant avec le rattachement à la nationalité, traditionnel en Pologne et dans de nombreux États du continent européen, et avec la division de la succession en meubles et immeubles, qui était traditionnelle en France, il a retenu en principe, sauf jeu de la clause d'exception ou choix par le défunt de sa loi nationale, le rattachement de l'ensemble de la succession à la loi de l'État dans lequel le défunt avait sa résidence habituelle au moment de son décès (art. 21).

Le règlement a également donné une précision importante concernant la réserve, en incluant celle-ci dans le domaine de la loi successorale.

\section{"Article 23}

\section{Portée de la loi applicable}

1. La loi désignée en vertu de l'article 21 ou 22 régit l'ensemble d'une succession.

2. Cette loi régit notamment:

[...]

h) la quotité disponible, les réserves héréditaires et les autres restrictions à la liberté de disposer à cause de mort ainsi que les droits que les personnes proches du défunt peuvent faire valoir à l'égard de la succession ou des héritiers; i) le rapport et la réduction des libéralités lors du calcul des parts des différents bénéficiaires;

$[\ldots]$ ".

Ces solutions sont bonnes et utiles, en particulier pour ce qui concerne la réserve. Le principe de l'unité de la succession a le grand avantage d'éviter les difficultés résultant de l'application de lois différentes à la réserve, suivant la nature mobilière ou immobilière des biens de la succession. La limitation de l'electio juris à la loi nationale du défunt limite la possibilité pour le testateur de choisir une loi qui lui permettrait de priver de toute part ses enfants ou son conjoint. Enfin la soumission de la réserve à la loi successorale donne une solution claire à la qualification de la réserve, institution successorale et non alimentaire pour le droit international privé de l'Union européenne. 
Aussi claires soient-elles, ces solutions ne résolvent pas toutes les questions, comme le montrent quelques développements récents.

Les difficultés tiennent moins au texte du règlement, qui est clair, qu'à la différence des législations des États membres sur l'existence même de la réserve.

Certains d'entre eux, comme indiqué, ignorent l'institution et lui sont hostiles. C'est le cas de l'Angleterre. Lord Collins of Mapusbury explique, dans le dernier supplément du Dicey-Morris, que la raison principale du refus du Royaume-Uni de participer à ce règlement est qu'il n'assure pas suffisamment la protection des bénéficiaires de libéralités inter vivos, en particulier les fondations et autres charities, contre le risque d'une action en réduction de ces libéralités - ce que les Anglais appellent le clawback - intentée par les réservataires en application de la loi successorale ${ }^{1}$.

D'autres États membres, les plus nombreux, s'inquiètent de l'éventualité que les héritiers, surtout si ce sont leurs nationaux, soient privés, en application de la loi successorale, de la part de réserve que leur accorde leur propre loi.

Actuellement en France on a deux affaires presque identiques, dont l'une défraie la chronique des réseaux sociaux depuis bientôt deux ans. Il s'agit de la succession du chanteur Johnny Hallyday, décédé en décembre 2017, laissant un testament américain léguant la totalité de ses biens à sa veuve, Lætitia, sans rien laisser à ses enfants nés d'unions précédentes et eux-mêmes artistes connus. La question litigieuse est de savoir si le défunt avait sa dernière résidence habituelle en France ou en Californie, ce qui déterminera la loi successorale et, dans l'hypothèse où ce serait la loi de Californie, si celle-ci ne heurte pas l'ordre public international français.

L'autre affaire, un peu plus ancienne mais non terminée, concerne la succession d'un autre artiste français, Maurice Jarre, compositeur de musique de cinéma, notamment des films Docteur Jivago et Lawrence d'Arabie, décédé en 2009. Lui aussi, qui vivait effectivement en Californie — donc pas de problème pour la loi applicable - avait déshérité ses enfants au profit de sa veuve, qui n'était pas la mère de ses enfants. Ces derniers avaient soutenu que la loi californienne, ignorant la réserve, était contraire à l'ordre public international français et des auteurs français assez nombreux étaient de cet avis. La Cour de cassation leur a donné tort par un arrêt du 27 septembre $2017^{2}$. Elle a posé la règle « qu'une loi étrangère désignée par la règle de conflit qui ignore la réserve héréditaire n'est pas en soi contraire à l'ordre public international français

${ }^{1}$ L.A. Collins, J. Harris : Dicey, Morris \& Collins. The Conflict of Laws, 5th Cumulative Supplement to the 15th ed. Sweet and Maxwell 2019, §27-136, p. 438.

${ }^{2}$ Civ. Civ., 27.9.2017, n 16-13151, AJ Famille, 2017, p. 595, note A. Boiché (Revue critique de droit international privé, 2018, p. 87, note B. Ancel). 
et ne peut être écartée que si son application concrète, au cas d'espèce, conduit à une situation incompatible avec les principes du droit français considérés comme essentiels".

La solution est raisonnable et avait déjà été recommandée. Il faut rappeler que la proposition de la Commission comportait une disposition selon laquelle l'ordre public ne pouvait pas être opposé à la loi successorale "au seul motif que ses modalités concernant la réserve héréditaire sont différentes de celles en vigueur dans le for" (art. 27 §), mais le règlement ne l'a pas reprise. On devait donc s'attendre à ce que les juridictions d'un État membre connaissant la réserve héréditaire soient un jour sollicitées d'opposer l'ordre public, dans un souci de protection des enfants ou du conjoint, aux lois étrangères ignorant celle-ci. Faire droit à une telle demande pourrait ruiner l'effectivité du règlement, car l'existence de la réserve ou son absence est un trait caractéristique du droit successoral de chaque État et l'application de l'exception d'ordre public retirerait beaucoup d'intérêt à la règle de conflit. De plus, cette application pourrait conduire à des solutions discriminatoires en fonction de la proximité de la situation avec le for et notamment de la nationalité des intéressés. A supposer que la loi successorale anglaise ignorant la réserve puisse heurter la sensibilité d'un juge français, il est probable qu'elle la heurterait davantage s'agissant de la succession d'un Français ayant eu sa dernière résidence habituelle à Londres que de celle d'un Anglais dans les mêmes conditions.

La solution moyenne adoptée par la Cour de cassation correspond bien mieux à l'esprit de l'exception d'ordre public, qui suppose un examen concret des circonstances concrètes du litige. Avant d'écarter la loi successorale ignorant la réserve, il faut examiner, cas par cas, si son application aboutirait à une situation inacceptable, en laissant par exemple sans ressources des enfants en bas âge ou en cours d'études. Déjà, la convention de La Haye du $1^{\text {er }}$ août 1989 avait prévu la possibilité d'une réserve permettant d'écarter la loi désignée par le défunt lorsque son application "priverait totalement ou dans une proportion très importante le conjoint ou l'enfant du défunt d'attributions de nature successorale ou familiale auxquelles ils auraient eu droit selon les règles impératives de la loi de l'État ayant fait cette réserve" (art. 24, $\S 1, \mathrm{~d}, 2$ ème tiret).

Pourtant, cette affaire Jarre n'est pas terminée. Les enfants déshérités n'ont pas accepté l'arrêt de la Cour de cassation. En août 2018, ils ont saisi la Cour européenne des droits de l'homme pour «manquement au respect des droits de la famille et pour atteinte excessive à notre sécurité juridique». La procédure est en cours à Strasbourg, mais il paraît douteux que l'on puisse reprocher une violation des droits de l'homme 
aux États qui organisent le règlement des successions sur des bases différentes de celles de l'État d'origine des plaignants.

Cette question précise a eu des retombées inattendues tout près de l'Union européenne et de la France, à Monaco $^{3}$. Ce pays a codifié son droit international privé par une loi du 28 juin 2017, préparée par une proposition de loi qui adoptait, en matière de succession, des dispositions proches de celles du règlement européen soumettant la succession à la loi du domicile sauf choix par le testateur de sa loi nationale, et incluant dans le domaine de la loi successorale la réserve et les restrictions à la faculté de disposer à cause de mort.

On s'est alors avisé que les héritiers d'un défunt monégasque domicilié à Londres pourraient être privés de la réserve prévue par le code civil monégasque et à l'inverse que les héritiers d'un Anglais domicilié à Monaco pourraient profiter de la réserve prévue par ce même code civil monégasque. Ces solutions ont été jugées inacceptables et la loi définitivement adoptée a introduit une disposition curieuse aboutissant en fait à soumettre la réserve à la loi nationale du défunt. Tout en maintenant l'inclusion de la réserve dans le domaine de la loi successorale, la loi a introduit une exception à la règle de l'application générale de la loi successorale. Le deuxième alinéa de l'article 63 dispose en effet « [t]outefois, il [le droit applicable à la succession] ne peut avoir pour effet de priver un héritier de la réserve que lui assure le droit de l'État dont le défunt a la nationalité au moment de son décès, ni d'appliquer la réserve à la succession d'une personne dont le droit de l'État dont elle a la nationalité au moment de son décès ne connaît pas ce régime». C'est une disposition bien étrange, car elle revient finalement à considérer l'application de la loi successorale monégasque à la succession d'un Anglais domicilié à Monaco comme contraire à l'ordre public de la Principauté. En réalité, l'article 63, al. 2 démembre l'unité de la loi successorale et tend à soustraire de son domaine la réserve héréditaire en en faisant une catégorie spéciale régie par la loi nationale du défunt.

Et, pour revenir à l'Europe et à notre règlement sur les successions, on peut faire la même observation sur le recours à l'exception d'ordre public en vue de retirer de la loi successorale les dispositions de celle-ci moins protectrices des intérêts des réservataires que celles de leur loi nationale. Car dans les droits qui la connaissent, la réserve représente rarement moins de la moitié de la succession et souvent plus des trois quarts.

${ }^{3}$ Loi n ${ }^{\circ} 1448$ du 22.6.2017, Journal de Monaco, n 8337, 7.7.2017, Revue critique de droit international privé 2018, p. 994. Sur cette loi, voir P. Lagarde : «La codification du droit international privé monégasque", Revue critique de droit international privé, 2018 , p. $753-774$. 


\section{Bibliographie}

Collins L.A., Harris J.: Dicey, Morris \& Collins. The Conflict of Laws, 5th Cumulative Supplement to the 15th ed. Sweet and Maxwell 2019, §27-136, p. 438.

Lagarde P.: "La codification du droit international privé monégasque». Revue critique de droit international privé, 2018, p. 753-774. 